\title{
Double layer propagation in experiments with electron beam injection
}

\author{
L. G. Bruskin ${ }^{1 *}$ and G. V. Khazanov ${ }^{2}$ \\ 'University of Tsukuba, Plasma Research Center, 1-1-1 Tennodai, Tsukuba 305, Japan \\ ${ }^{2}$ University of Michigan, Space Physics Research Laboratory, Ann Arbor, MI 48109, U.S.A.
}

Reccived in final form 13 November 1992

\begin{abstract}
Electron beam injection into a plasma is investigated using the analytical inverted BernsteinGreen-Kruskal method. Particle number and momentum conservation laws are applied to evaluate the propagation velocity and potential drop on the leading edge of the beam. Electric potential is supposed to be monotonic, thus the leading front has a double-layerlike structure. For the case of cold particles, analytical expressions for the double layer velocity and potential drop are obtained. It is pointed out that double layer velocity differs from the initial electron speed: even for weak beams a noticeable deceleration takes place. Strong beams are found incapable of penetrating into plasma-their propagation velocity is very small. Ambient electrons undergo a considerable acceleration forming a return current which neutralizes the injector. Possible instability of the distribution functions is discussed.
\end{abstract}

\section{Introduction}

Investigation of the plasma response to electron or ion beam injection is of interest with regard to active experiments in space. Such experiments, being held already for more than 20 years, are designed to study the fundamental laws of plasma physics as well as to help understand the natural processes occurring in the upper atmosphere and magnetosphere: auroral arcs, parallel electric fields and currents. electron beam acceleration, etc. Recently, heam injections were applied as a part of more complicated experiments, such as tethers in space. pulsing jets, plasma instability excitation or artificial magnetosphere creation projects.

Although there are various kinds of active experiments being held in space or desiring to be held there in the future. nearly all of them have much in common-they start suddenly, which leads to an eruption-like modi-

* On leave of absence from Irkutsk Polytechnic Institute. Department of Mathematics, 83 Lermontov S1., Irkutsk. Russia. Correspondence to: $\mathrm{G}$. V. Khazanov fication of the ambient plasma. The results of such an influence are various front formations: gydrodynamic fronts, collisionless shocks, turbulent shock waves, ionacoustic waves, etc.

The double layer (DL) is one of the types of front which seems to appear in very different situations. both in laboratory and natural plasmas. The traditional definition supposes double layers to be a potential drop in a constant-density plasma with a monotonic potential distribution in it. DLs in space physics arc a subject of interest because of their capability of accelerating electrons along the magnetic field lines. Block (1972) was the first to assume DLs to be the main reason for accelerated particles in a collisionless plasma.

Later experiments discovered that double layers are quite different. They may obey the traditional definition or may not. They may exist in stable and non-stable plasmas. they may separate two different plasmas, they may have a shock-wave-like structure with non-monotonic potential rise, they may be moving or resting. From the very first works. DLs were supposed to exist in a current carrying plasma. They were first discovered in electric discharges in tubes containing mercury vapour. Langmuir (1929) supposed that the steep vapour glow changes in the tubes were caused by a potential drop near the cathode which was nearly equal to the external power applied.

Since that time. DLs have been found in a large number of laboratory experiments. Lutsenko et al. (1976) pointed out that moving double-layer type structures formed in a current carrying plasma when the current density exceeded its threshold value. They observed also other phenomena accompanying double layer propagation: potential and current oscillation, acceleration of electrons, plasma turbulence excitation and recurring formation of double layers. Similar results were observed by Leung $e t$ al. (1980). Torven (1978). Iizuka et al. (1979) and Saeki et al. (1980). Electric field measurements in space plasma (Boehm and Mozer, 1981; Falthammer, 1978) founded strong parallel electric fields existing in relatively small regions, which were interpreted as moving double layers.

But the DL problem is of interest also because of its connection with theories of collisionless shocks, anomalous resistivity, ion-acoustic turbulence etc. That is why 
DLs were studied extensively both by analytical and numerical methods. It was pointed out that DLs cannot only accelerate particles: they lead also to electron and ion reflection on both sides (Artsimovitch and Sagdeev, 1979). The formation of a plasma density cavity with a space charge was studied in Singh's $(1980,1982)$ numerical investigation, as well as the effects of recurring appearance and propagation of DLs. Schamel and Bujarbarua (1983) pointed out that DLs may be classified as two types: weak (with size much more than both Debye length / and gyroradius of the particles. so that gydrodynamic theory might be applied) and strong (with a potential drop width of about several $/$ ). The theory of weak DLs was developed by Schamel and Bujarbarua (1983). Goswami et al. (1986) and Sutradhar and Bujarbarua (1988) who investigated ion and electron-hole formation for different situations and conditions.

Strong DLs are the subject of much more interest in this paper because they are likely to exist in all types of active experiments mentioned above. The greatest troubles in the problem discussed are the plasma processes leading to formation of a structure with a potential drop. Some authors involve Buneman instability, Pierce instability or rarefaction instability (Iizuka et al., 1979; Smith and Goertz, 1978: Carlquist, 1972). On the other hand, other authors came to the conclusion that instabilities are not responsible for the formation of double layers. They claimed that double layers developed because of the appropriate particle distributions. It was noticed by Singh (1982) that our understanding of the physical processes during the formation period is not yet complete.

In the present paper. we will not be concerned with this question. The problem of stability of DLs will not be handled here. either. Our purpose is to obtain quantitative analytical expressions for particle distribution, electric potential structure and propagation velocity of double layers forming in active experiments. Despite the fact that DLs have been studied extensively over the last few years. the fundamental question about their parameters is not vet solved completely. A comprehensive study was provided only by numerical analysis (Singh, 1982: Singh and Hwang. 1988: Okuda ('t al.. 1987). Some properties of double layers were examined by Gurevich et al. (1985) for the case of a stationary (resting) double layer in plasma where electrons and ions are injected from different sides. In this case, the charged particle concentration was found as a function of potential $\Psi$. but the potential distribution was not derived. It was pointed out that resting DI.s may exist only for specially selected distribution functions of injected ions and electrons. In Section 2 we shall extend their research in order to obtain the potential and velocity of the moving DL for arbitrary distribution functions with arbitrary parameters.

DLs. solitary and ion-acoustic waves were studied by Schamel (1972) who had proposed the inverted BernsteinGreen-Kruskal method to solve this problem. According to this method. all distribution functions are prescribed so that the potential can be easily evaluated from the Poisson equation. In order to get a solitary wave-like profile. Schamel supposed all free distribution functions to be the same on both sides of the wave, which may only be shifted due to potential variation. Of course. this suggestion is not valid for beam injection, where plasmas separated by the shock front are quite different.

A very simple method for yielding the potential drop and front velocity was proposed by Lyatsky (1981). Assuming the double layer to be strong enough to reflect all the injected electrons, he derived DL parameters using only particle number and energy conservation laws. Although this technique can be applied only to strong beams, it seems to be very promising because conservation laws might be useful for arbitrary cases as well. They will be taken in Section 2 to derive unknown constants in the DL solution. In Section 3 we point out that the Lyatsky solution is an asymptotic form of our expressions in the cold particles limit.

In Section 4, the results of our investigation and possible conclusions are discussed.

\section{Electron beam injection}

Consider an electron beam propagating along the magnetic field lines in a collisionless ambient plasma. Electric charge insertion will lead to a potential drop appearance at the leading front of the beam. To recover quasineutrality, some of the ambient electrons should be accelerated by this drop in the direction opposite to beam velocity. Of course ambient ions are also affected by the moving beam front, but their velocity will increase by $\sqrt{m_{\mathrm{i}} / m_{\mathrm{e}}}$ times less than that of the electrons. We conclude that quasineutrality is recovered due to ambient electrons' acceleration rather than that of ions, but this does not mean that plasma ions may not be taken into account. During the front passage they acquire the same energy as the electrons and the electric field accelerates them in the beam movement direction. In order to obey the energy conservation law. it is necessary to calculate the beam energy decrease due to acceleration of both ions and electrons, but the ambient ion concentration decreases negligibly as long as the beam density is not too large. If the plasma is dense enough, it leads to insufficient ambient electron acceleration to compensate for the beam charge. For another case, the potential drop must be high, as well as the velocity of the accelerated electrons. This means a beam energy decrease and its deceleration as a consequence.

Discussing this problem in the reference frame associated with the moving front we can conclude the following.

(1) There are two types of injected electrons moving from the left side (see Fig. la):

(a) electrons with energy greater than the potential drop (free electrons capable of travelling above the electrostatic barrier): and

(b) electrons which cannot jump over the barrier. These will be turned back by the front. We shall call them reflecting.

(2) There are two types of ambient ions moving from the right side (see Fig. Ib) :

(a) decelerated free ions capable of jumping the potential barrier: and

(b) reflecting ions which change their velocity to the opposite direction. 

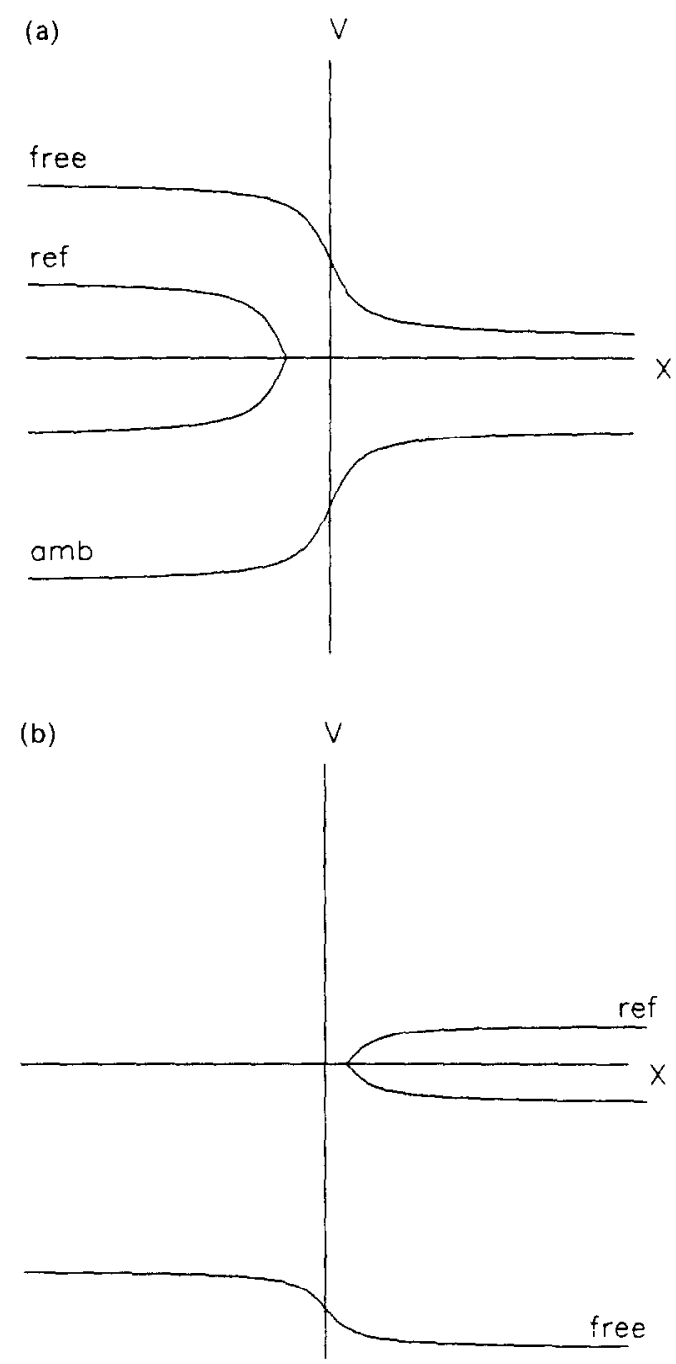

Fig. 1. (a) Free, reflecting and ambient electron velocity. The DL is located at the $x=0$ point. (b) Free and reflecting ion velocity

We assume the potential to be monotonic, as in the other case a third class of particles would appear : trapped particles in a potential well. We suppose all distribution functions to be Maxwellian far from the double layer. In a frame of reference associated with a moving front. ambient electrons and ions have the same drift velocity in the $x$-direction.

The set of equations describing the plasma components in an electrostatic wave is the Vlasov equation for each particle species, the Poisson equation and the quasineutrality condition. The solution technique for the problem of such a kind was developed by Schamel (1972). inverting the method given by Bernstein. Green and Kruskal (1957). According to Schamel, all distribution functions are prescribed and the potential must be sought. All distribution functions have to obey physically sensible conditions-they must depend on the constants of motion for the Vlasov cquation. In our case it means that the distribution function is a function of energy, that is a sum of kinetic and electrostatic energy in the field of the double layer. For example, a distribution function of accelerated ambient electrons may be chosen by replacing the velocity $v$ in the initial Maxwellian func- tion with $\sqrt{t^{2}-2 e} \bar{\Psi} / m_{\mathrm{e}}$, because all the particles with velocities from $v$ to $v+\mathrm{d} v$ were initially distributed near $\sqrt{r^{2}-2 e \Psi / m_{c}}$. Their energy is thus conserved.

For ambient, free and reflecting electrons, the set of prescribed distribution functions at an arbitrary point may be written as follows:

$$
\begin{aligned}
& f_{x}=\frac{k_{0}}{\sqrt{\pi r_{\mathrm{T}}}} \exp \left[-\left(-\sqrt{r^{2}-\psi}-r_{0}\right)^{2}\right] ; \\
& -x<t<-\sqrt{\psi} \\
& f_{\mathrm{f}}=\frac{k_{1} \sqrt{\alpha}}{\sqrt{\pi l_{\mathrm{T}}}} \exp \left[-\alpha\left(\sqrt{v^{2}+\psi_{0}-\psi}-v_{1}\right)^{2}\right] ; \\
& \sqrt{\psi}<t<x \\
& f_{1}=\frac{k_{1} \sqrt{\alpha}}{\sqrt{\pi r_{T}}} \exp \left[-\alpha\left(\sqrt{v^{2}+\psi_{0}-\psi}-v_{1}\right)^{2}\right] ; \\
& -\sqrt{\psi}<t<\sqrt{\psi} .
\end{aligned}
$$

In the above equations, $v_{\mathrm{T}}=\left(2 T_{\mathrm{e} 0} / m_{\mathrm{e}}\right)^{1 / 2}$ is the thermal velocity of ambient electrons, $v$ is the velocity in units of $\varepsilon_{\mathrm{T}}, \psi$ is electrostatic potential normalized by $T_{\mathrm{e} 0} / e, \psi_{0}$ is the unknown maximum value of $\psi, v_{0}$ is the drift velocity of the free electrons (it is negative, $-v_{0}$ is equal to the double layer velocity in the laboratory reference frame), $v_{1}$ is the drift velocity of the free electrons $\left(-v_{1}\right.$ is the drift velocity of the reflecting particles, $v_{1}$ derives the beam drift velocity in the laboratory reference frame $v_{\mathrm{h}}=v_{1}-v_{0}$ ). Both $v_{1}$ and $v_{0}$ are normalized by $v_{\mathrm{T}}, \alpha$ is the ratio of ambient electron temperature to beam temperature. Constants $k_{0}, k$, will be determined from boundary conditions introducing the concentration of injected particles on the left side $n_{\text {(ini }}$ and unperturbed electron density of the medium moving from the right side $n_{0 \text { amb }}$. The last constant is the concentration of particles achieving the double layer: in the case when $v_{\mathrm{T}} \ll\left|v_{0}\right|$, nearly all the electrons will overcome the front.

Ambient electrons located at the point with potential $\psi$ are already accelerated, so their velocities may vary in the range $-x<t<-\sqrt{\psi}$. The velocity of the other species can be derived the same way, as is pointed out in equations $(1 \mathrm{a}-\mathrm{c})$.

The densities which are given by integration of the distribution function cannot he expressed by the ordinary functions. However all of them might be reduced to an crror function or to a new function:

$$
\Phi(x, y, z)=\frac{1}{\sqrt{2}} \int_{=}^{x} \frac{t}{\sqrt{t^{2}+x}} \exp \left[-(t+y)^{2}\right] \mathrm{d} t .
$$

This function can easily be obtained by solving the integral numerically or by approximation for the different values of the arguments.

Thus, all the electron densities are expressed as follows:

$$
\begin{gathered}
n_{x}=k_{0} \Phi\left(\psi, v_{0}, 0\right), \\
n_{\mathrm{f}}=k_{1} \Phi\left(\alpha\left(\psi-\psi_{0}\right),-\sqrt{\left.\alpha v_{1}, \sqrt{\alpha \psi_{0}}\right),}\right. \\
n_{r}=2 k_{1}\left\{\Phi\left[\alpha\left(\psi-\psi_{0}\right),-\sqrt{\alpha v_{1}}, \sqrt{\alpha\left(\psi_{0}-\psi\right.}\right)\right] \\
\left.-\Phi\left[\alpha\left(\psi-\psi_{0}\right),-\sqrt{\alpha v_{1}}, \sqrt{\alpha \psi_{0}}\right]\right\} .
\end{gathered}
$$


The boundary conditions yield:

$$
k_{0} \simeq n_{\text {oamb }}, \quad k_{1} \simeq n_{\text {onij. }} .
$$

We assumed here $v_{T} \ll\left|v_{0}\right|$.

Distribution functions of the ambient ions (free and reflecting) reaching the double layer from the right side (see Fig. 1b) can be obtained in the samc way:

$$
\begin{gathered}
F_{\mathrm{f}}=\frac{k_{2}}{\sqrt{\pi u_{\mathrm{T}}}} \exp \left[-\beta\left(-\sqrt{u^{2}+\gamma \psi}-v_{0}\right)^{2}\right] ; \\
-\propto<u<-\sqrt{\gamma\left(\psi_{0}-\psi\right)}, \\
F_{\mathrm{r}}=-\frac{k_{2}}{\sqrt{\pi u_{\mathrm{T}}} \exp \left[-\beta\left(\sqrt{u^{2}+\gamma \psi}+v_{0}\right)^{2}\right],} \\
-\sqrt{\gamma\left(\psi_{0}-\psi\right)}<u<\sqrt{\gamma\left(\psi_{0}-\psi\right)} .
\end{gathered}
$$

New parameters are defined by:

$$
u_{\mathrm{T}}=\left(2 T_{\mathrm{i}} / m_{\mathrm{i}}\right)^{12,}, \quad \beta=v_{\mathrm{T}}^{2} / u_{\mathrm{T}}^{2}, \quad \gamma=m_{\mathrm{e}} / m_{\mathrm{i}},
$$

where $u$ is the ion velocity normalized by $v_{\mathrm{T}}$ and $T_{\mathrm{i}}$ is the ion temperature.

Integrating $F$ over $u$ yields ion concentration as a function of $\psi$ :

$$
\begin{gathered}
c_{\mathrm{f}}=k_{2} \Phi\left(-\gamma \beta \psi, \sqrt{\beta} v_{0}, \sqrt{\gamma \beta \psi_{0}}\right), \\
c_{\mathrm{r}}=2 k_{2}\left[\Phi\left(-i \beta \psi, \sqrt{\beta} v_{0}, \sqrt{\gamma \beta \psi}\right)\right. \\
\left.-\Phi\left(-\gamma \beta \psi, \sqrt{\beta} v_{0}, \sqrt{\gamma \beta \psi_{0}}\right)\right] .
\end{gathered}
$$

$k_{2}$ is defined by the boundary condition:

$$
k_{2}=k_{n}=n_{0 \text { amb. }}
$$

In order to get general solutions $n(x), c(x), \psi(x)$ we ought to integrate Poisson`s equation:

$$
\begin{gathered}
\frac{\partial^{2} \psi}{\partial x^{2}}=-4 \pi e(c-n), \\
c=c_{\mathrm{r}}+c_{\mathrm{r}} . \quad n=n_{x}+n_{\mathrm{b}}+n_{\mathrm{r}},
\end{gathered}
$$

with properties:

$$
\psi(-x)=\psi_{1} . \quad \psi(x)=0 .
$$

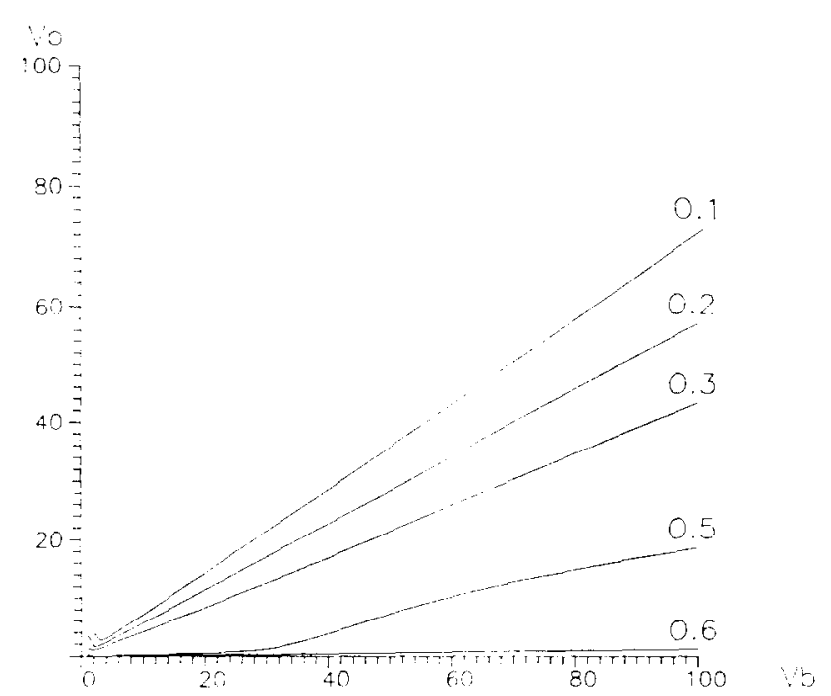

Fig. 2. DL velocity as a function of beam injection velocity

$$
c(-\infty)=n(-\infty), \quad c(\infty)=n(\infty) .
$$

It usually leads to the same results : violation of the quasineutrality condition and electron cavity formation (Singh. 1982; Gurevich. 1985) inside the double layer (the obtained width of DL used to be of the order of $\hat{i}_{D}$ ). However, we do not need to solve Poisson's equation if we are interested only in obtaining all the functions outside the double layer. When $|x| \gg \lambda_{D}$, the concentrations of the species obey the electric neutrality condition. The second relation is a consequence of the momentum conservation law. It represents the equality of momentum flux (or pressure equality) on the left and the right side of the DL.

On the left boundary, the ncutrality condition has the form :

$$
\begin{array}{r}
k_{0} \Phi\left(\psi_{0}, v_{0}, 0\right)+\frac{k_{1}}{2} \operatorname{erfc}\left[\sqrt{\alpha}\left(\sqrt{\psi_{0}}-v_{\mathrm{b}}-v_{0}\right)\right] \\
+k_{1}\left\{\operatorname{erf}\left[\sqrt{\alpha}\left(\sqrt{\psi_{0}}-v_{\mathrm{b}}-v_{0}\right)\right]+\operatorname{erf}\left[\sqrt{\alpha}\left(v_{\mathrm{b}}+v_{0}\right)\right]\right\} \\
=k_{1} \Phi\left(-\gamma \beta \psi_{0}, \sqrt{\left.\beta v_{0}, \sqrt{\gamma} \beta \psi_{0}\right),}\right.
\end{array}
$$

where $v_{\mathrm{b}}$ means the beam drift velocity in a laboratory frame of reference.

Here, the connection between our function $\Phi$ and the error function was taken into account :

$$
\Phi(0, y, z)=\operatorname{erfc}(z+y) .
$$

Since we expect the concentration of reflecting ions and thus free electrons to be negligible, the neutrality condition on the right boundary cannot provide any information about DL parameters.

The momentum flux (or pressure) equality yields:

$$
\begin{aligned}
& k_{1} \sqrt{x} \int_{, \bar{\psi}}^{x} \exp \left[-\alpha\left(|v|-v_{1}\right)^{2}\right] r^{2} \mathrm{~d} v
\end{aligned}
$$

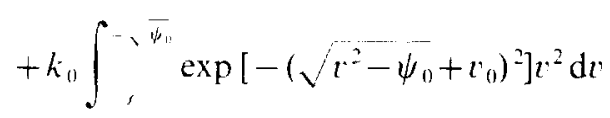

$$
\begin{aligned}
& +\frac{k_{0} \beta}{\gamma} \int^{0} \exp \left[-\beta\left(\sqrt{u^{2}+\gamma \psi_{0}}+v_{0}\right)^{2}\right] u^{2} \mathrm{~d} u \\
& =k_{0} \int_{x}^{\prime \prime} \exp \left[-\left(|v|+v_{0}\right)^{2}\right] r^{2} \mathrm{~d} v \\
& +k_{\sqrt{ }} \sqrt{\alpha} \int_{0}^{1} \exp \left[-\alpha\left(\sqrt{v^{2}}+\psi_{0}-v_{1}\right)^{2}\right] v^{2} \mathrm{~d} v \\
& +\begin{array}{c}
k_{i n} \sqrt{\beta} \\
\vdots
\end{array} \int_{,}^{-\cdots w} \exp \left[-\beta\left(|u|+v_{0}\right)^{2}\right] u^{2} \mathrm{~d} u .
\end{aligned}
$$

Because of the choice of distribution functions, depending on the motion constants, the energy conservation law is also justified.

Relationships (5) and (6) represent the solution of our problem in that they derive both unknown constants $v_{0}$ and $\psi_{1}$. In the general case they should be solved numerically; the results of computer investigation of equations (5) and (6) will be discussed in Section 4. But, for a very important case of cold particles. these equations can be reduced yielding analytical expressions for $v_{0}$ and $\psi_{0}$. 


\section{Cold particles approximation}

If all the drift velocities $\left(v_{0}, v_{\mathrm{b}}, v_{0}+v_{\mathrm{b}}\right)$ are much greater than the thermal speed of electrons (or if the temperature of each species is negligible in comparison with its drift kinetic energy), the function $\Phi$ and all the integrals in equations (5) and (6) can be reduced using the asymptotic Laplace method. For the case $v_{0}+v_{\mathrm{b}}<\sqrt{\psi_{0}}$, our equations can be written as follows:

$$
\begin{gathered}
2 k_{1} \quad \frac{k_{0} v_{0}}{\sqrt{v_{0}^{2}+\psi_{0}}}=k_{0}, \\
2 k_{1}\left(v_{h}+v_{0}\right)^{2}-k_{0} v_{0}\left(\sqrt{r_{0}^{2}+\psi_{0}}+v_{0}\right) \\
=\frac{k_{0} v_{0}^{2}}{\gamma}\left(1-\sqrt{1-\frac{\gamma \psi_{0}}{r_{0}^{2}}}\right) .
\end{gathered}
$$

The case when $v_{0}+v_{\mathrm{b}}>\sqrt{\psi_{0}}$ is outwith our interest. because it means a considerable density of free electrons and reflecting ions on the right boundary. The potential drop cannot be so large as to reflect a noticeable amount of heavy ions. Taking into account relation $\gamma \ll 1$ and substituting $\sqrt{v_{0}^{2}+\psi_{0}}=t$, we obtain an analytical solution of equations (7) and (8):

$$
\begin{aligned}
& v_{0}=-v_{\mathrm{b}}\left(1-2 \frac{k_{1}}{k_{0}}\right)\left(1-2 \frac{k_{1}}{k_{0}}+\sqrt{\frac{k_{1}}{k_{0}}}\right)^{-1}, \\
& \psi_{01}=4 r_{\mathrm{b}}^{2} k_{1}\left(k_{0}-k_{1}\right)\left(1-2 \frac{k_{1}}{k_{0}}+\sqrt{\frac{k_{1}}{k_{0}}}\right)^{-2} .
\end{aligned}
$$

These expressions coincide with the solution given by Lyatsky (1981). He supposed these relations to be valid in a strong double layer limit when the assumption $k_{1} \ll k_{0}$ is not justified. Now we see that they are still valid for weak double layers as well if only the drift velocity of each species is much larger than its thermal speed.

If $k_{1} \simeq 0.5 k_{0}$, then as follows from equation (9) $k_{0} \rightarrow 0$. It means that the approximation for cold particies becomes invalid. In this case, the thermal speed should be taken into account to obtain reasonable non-zero values for DL propagation velocity.

\section{Discussion}

We have developed here an analytical model of beam injection from a spacecraft into an ambient plasma and hatve oblained an asymptotic solution in the cold particles approximation. We have pointed out that double layer velocity and potential drop depend on initial beam parameters in a rather complicated way, not only through beam current $n_{t, n i} t_{h}$. If beam density is fixed, the DL propagation velocity $v_{n}$ is proportional to injection speed $i_{h}$. This conclusion is confirmed either by relation (9) and by the results of numerical solution of nonlinear equations (5) and (6), which are shown in Fig. 2. (All the results plotted below correspond to $\alpha=1$, ion parameters correspond to $\mathrm{O}^{+}$ions.)

For the case when beam density $n_{\text {0ini }}<0.4 n_{\text {Uamb }}$, the linear approximation becomes invalid when injection vel- ocity is smaller than $\simeq 2 i_{\mathrm{T}}$. When $n_{0 \mathrm{inj}} / n_{0 \mathrm{amb}}>0.4$, the linear approximation is violated even for $t_{\mathrm{b}} \simeq 10-20$.

Potential drop is proportional to $l_{\mathrm{b}}^{2}$, while beam density does not exceed half of the ambient density, in good agreement with Singh and Hwang's (1988) result.

If $v_{\mathrm{b}}$ is fixed the asymptotic solution exists only while $n_{0 \mathrm{nin}}<0.5 n_{0 \mathrm{umb}}$, when the cold particles approximation is justified. Examining the expression (9) we can expect a considerable decrease of $v_{0}$ when the $k_{1} / k_{0}$ ratio approaches 0.5 . Such a decrease really takes place, as follows from numerical calculations plotted out in Fig. 3. When $v_{\mathrm{b}}<30$, the double layer velocity becomes negligible for $k_{1} / k_{0} \simeq 0.5$, and when $k_{1} / k_{0}>0.6$, the DL does not propagate into the ambient plasma for all values of initial beam energy. In this case, the double layer must be more like a sheath in the vicinity of a spacecraft and the strong beam must be returned to the vehicle inside that sheath. Numerical solution of the Vlasov and Poisson equations carried out by Singh and Hwang (1988) and particle modelling (Okuda et al., 1987) pointed out that an electron beam indeed cannot penetrate into plasma if the ratio $k_{\mathrm{j}} / k_{0}$ is about 0.5 . When the electron beam is weak enough, the propagation velocity is a bit less than $v_{0}$, thus beam deceleration always takes place.

Figures 4 and 5 show the distribution function behind the DL for the cases $v_{0}=10, k_{1} / k_{0}=0.3$ and $v_{0}=100$. $k_{1} / k_{0}=0.5$. Both figures demonstrate that nearly all the initially injected electrons were reflected by the DL. It means that the potential drop is higher than the kinetic energy of the electrons in a reference frame associated with the moving double layer. However, it does not mean that beam electrons are definitely returned back by the DL to provide return current towards the spacecraft. Their velocity acquired after reflection, $v=-v_{\mathrm{b}}+2\left|v_{0}\right|$, may remain positive as it happens for $k_{1} / k_{0} \leqslant 0.25$.

Distribution functions of free and reflected electrons are symmetric with a centre positioned at the $-r_{0}$ point. The third part of the distribution function corresponds to electrons of the ambient plasma. They undergo a considerable acceleration towards the injector, so their aver-

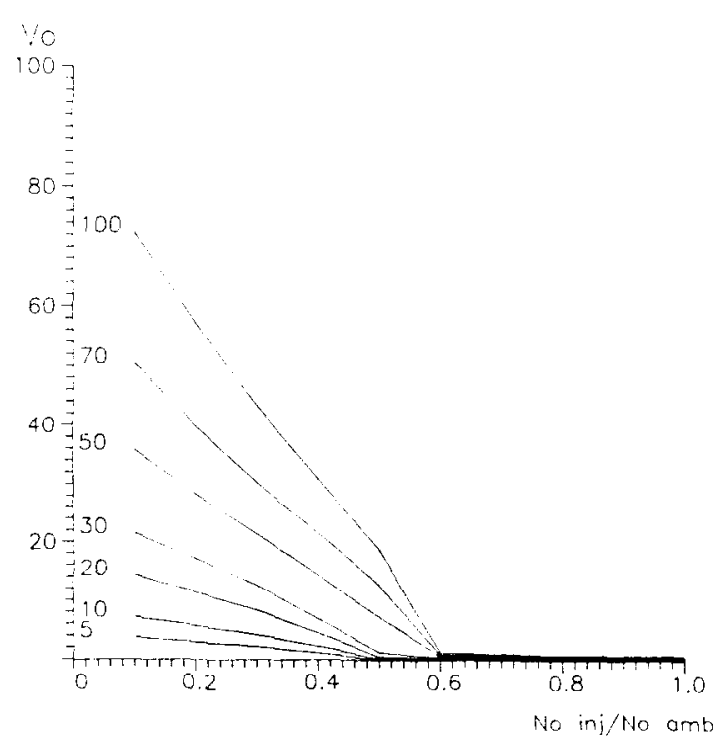

Fig. 3. DL velocity as a function of beam density 


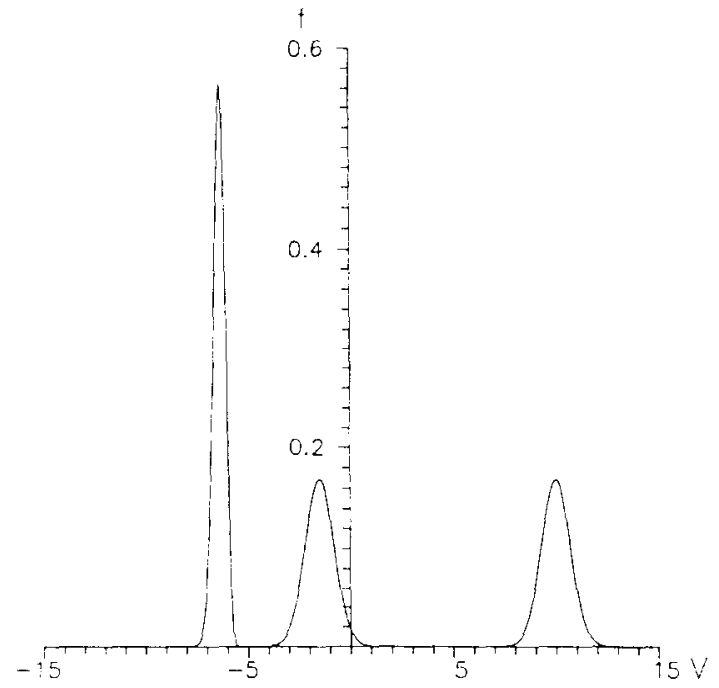

Fig. 4. Distribution function behind the double layer, $v_{0}=10$, beam to ambient electron density ratio $k_{1} / k_{0}=0.3$

age velocity is about $-\sqrt{v_{0}^{2}+\psi_{0}}$. In all cases, the double layer forms a secondary beam which provides a return current neutralizing the injector. This current may consist mainly of accelerated ambient electrons (Fig. 4) or of reflected beam particles (Fig. 5).

Although the kinetic energy of injected electrons is much higher than their temperature, one can notice that the width of the distribution function (i.e. effective thermal speed) appears to be larger than the beam drift velocity. This effecl was described by Singh and Schunk (1983). It takes place due to counterstreaming beams existing on the high potential side of the double layer. Of course, effective temperature increase does not mean any transformation of drift kinetic energy.

The distribution function plotted in Figs 4 and 5 cannot be stable. Strong beam-plasma interaction behind the double layer will occur due to counterstreaming beams. As a possible result of this interaction, narrow peaks in the distribution function may become broader, forming plateau-like tails. It really happened in Singh and Hwang`s (1988) and Okuda et al. 's (1987) numerical models.

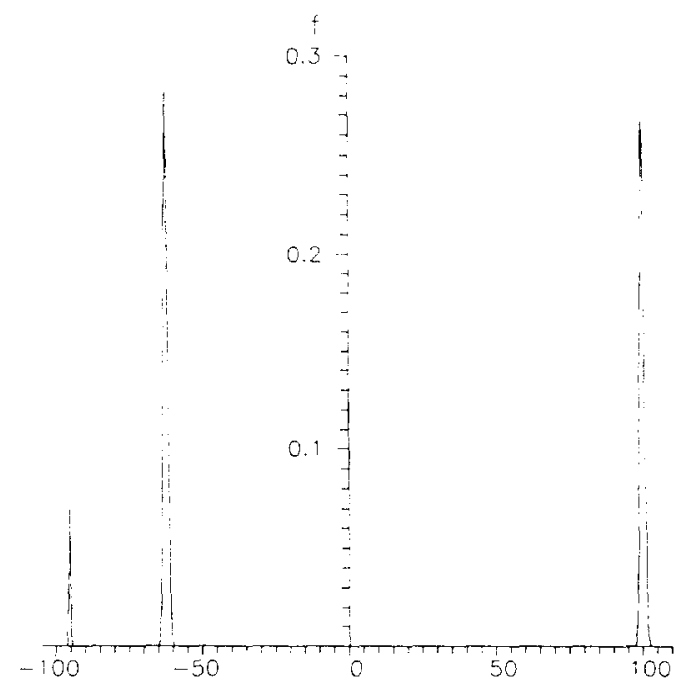

Fig. 5. Distribution function for $t_{0}=100, k_{1} / k_{0}=0.5$
The potential drop $\psi_{0}$ evaluated in this paper is connected with the spacecraft potential with respect to ambient plasma. Vehicle potential determination is a rather complicated problem which includes charging of the surface at early times, collection of the ambient electrons by the whole surface and development of a self-consistent electric field. In this respect, the problem becomes threedimensional and non-stationary. But, in steady injection (for $t \gg \omega^{-1}$, where $a$ is electron-plasma frequency), potential oscillations can be neglected. Assuming the neutralization process to be one-dimensional along the magnetic field lines, we can identify the potential drop in a double layer with an average spacecraft potential with respect to a plasma.

It is likely to be an overestimation because of two reasons. The first is the assumption with regard to one dimension. The second reason is concerned with possible beam-plasma instability which leads to electron diffusion in a phase space towards the low velocities. Reduction of average energy behind the double layer corresponds to potential decrease. Thus, the potential of the spacecraft in an active experiment must be smaller than can he evaluated from equation (10).

Another limitation of our analytical model is connected with the assumption of monotonic potential $f$. However, numerical simulations show that electric potential may be monotonic [in structures like electron shocks simulated by Singh and Schunk (1983, 1984), Singh and Hwang (1988)] and may have oscillating distributions [in double layers forming in response to the injection of moderate beams inlo a space plasma; Singh and Hwang (1988)]. Both structures have much in common since they form due to fast-propagating energetic electrons in unperturbed plasma, while ambient ions remain nearly immobile. Both cases differ from double layers with virtual cathode simulated by Singh and Schunk (1984), where ion motion is of importance.

For the case of beam injection where potential distribution was found to be non-monotonic, the amplitude of oscillations was significantly smaller than the potential step magnitude. Our model also corresponds to propagation of collisionless electron shocks, thus for the purposes of analytic simulation we can assume a steep monotonic variation of electric potential. More comprehensive study of the potential structure leads to a Poisson equation investigation inside the double layer, taking into account not only free and reflecting particles, but also trapped electrons in the potential well. These should be the subject of further analytical modelling of electron beam injections.

Acknowledgements. This work was supported by NASA Grant Nos. NAGW-1619 and NAG5-1500 as well as NSF Grant No. ATM-9114409.

\section{References}

Artsimovitch, L. A. and Sagdeev, R. Z., Plasma Physics for Physicists, Nauka, Moscow, 1979.

Bernstein, I. B., Green, J. M. and Kruskal, M. D., Phys. Rev. 108, 546, 1957.

Block, L. P., Potential double layers in the ionosphere, Cosmic Electrodyn. 3, 349, 1972. 
Boehm, M. N. and Mozer, F. S., An S3-3 search for confined region of large parallel electric fields, Geophys. Res. Lett. 8, 607,1981

Carlquist, P., Cosmic Electrody'n. 3, 337, 1972.

Falthammer, C. G., General mechanisms of magnetic field aligned electric field in the magnetosphere, J. Geomag. Geoelect. 30, 419, 1978.

Goswami, K. S., Kalita, M. K. and Bujarbarua, S., Theory of small amplitude electron acoustic double layers, Plasma Phys. Contr. Fusion 28, 289, 1986.

Gurevich, A. V., Meerson, B, I. and Rogachevcky, I. V., Kinetic theory of stationary double layer in plasma, Societ J. Phys. Plasma 11, 1213, 1985.

lizuka, S., Saeki, K., Sato, N. and Hatta, Y., Buneman instability. Picrce instability and double layer formation in collisionless plasma, Phys. Rev. Lett. 43, 1404, 1979.

Langmuir, I., The interaction of electron and positive ion space charges in cathode sheaths, Phys. Ret. 33, 954, 1929.

Leung, P., Wong, A. V. and Quon, B. H., Formation of double layers, Phys. Fluids 23, $992,1980$.

Lutsenko, E. I., Sereda, N. D. and Kontsevoy, L. M., Investigation of space charge formation in plasma, Sotiet J. Phys. Plasma 2, 72, 1976.

Lyatsky, V. B., Electron beam movement in a cold plasma, Gcomagn. i acronomiya 21, 378. 1981.
Okuda, H., Horton, R., Ono, M. and Ashour-Abdalla, M., Propagation of a nonrelativistic electron beam in a plasma in a magnctic ficld, Phys. Fluids 30, 200, 1987.

Saeki, K., Iizuka, S. and Sato, N., Ion heating due to double layer disruption in a plasma. Phys. Res. Lett. 45. 1853. 1980.

Schamel, H., Stationary solitary, soidal and sinusoidal ion acoustic waves, Plasma Phys. 14, 905, 1972.

Schamel, H. and Bujarbarua, S., Analytical double layers, Phys. Fluids 26, 190, 1983.

Singh, N.. Computer experiment on the formation and dynamics of electric double layers, Plasma Phys. 22, 1, 1980.

Singh, N., Double layer formation, Plasma Phys. 24, 639, 1982.

Singh, N. and Hwang, K. S.. Electric potential structures and propagation of electron beam injected from a spacecraft into a plasma, J. geophys. Res. 93, 10,035, 1988.

Singh, N. and Schunk, R, W., Collisionless electron shocks in electron beam-plasma systems, Phis. Fluids 26, 2781, 1983.

Singh, N. and Schunk, R. W., Plasma response to the injection of an electron beam, Plasma Phys. Contr. Fusion 26, 859 . 1984.

Smith, R. A. and Goertz, C. K., J. geophys. Res. 83, 2617, 1978.

Sutradhar Das, S. and Bujarbarua, S., Formation of ion acoustic double layers, J. Phys. Soc. Japan 57, 499, 1988.

Torven, S., Current limitation by an electric double layer in ion laser discharges, J. appl. Phys. 49, 2563, 1978. 